PROCEEDINGS OF THE

AMERICAN MATHEMATICAL SOCIETY

Volume 137, Number 11, November 2009, Pages 3703-3708

S 0002-9939(09)10030-8

Article electronically published on June 25, 2009

\title{
HARNACK'S INEQUALITY FOR VISCOSITY SOLUTIONS TO DEGENERATE ELLIPTIC EQUATIONS IN TWO VARIABLES
}

\author{
G. GRIPENBERG
}

(Communicated by Matthew J. Gursky)

\begin{abstract}
A Harnack inequality is established for viscosity solutions to the elliptic equation $F\left(\mathbf{x}, u, D u, D^{2} u\right)=0$ in two variables.
\end{abstract}

\section{Introduction AND StATEMEnt of RESUlts}

The purpose of this paper is to establish a Harnack inequality for viscosity solutions to the equation

$$
F\left(\mathbf{x}, u, D u, D^{2} u\right)=0, \quad \mathbf{x} \in \Omega \subset \mathbb{R}^{2},
$$

under weaker assumptions than uniform ellipticity on $F$ (which requires that $\lambda\|N\|$ $\leq F(\mathbf{x}, r, \mathbf{p}, M)-F(\mathbf{x}, r, \mathbf{p}, M+N) \leq \Lambda\|N\|$ when $N \geq 0$ with $0<\lambda \leq \Lambda$; see 2 , p. 12] where the equation $-F=0$ is considered). Unfortunately this is only done in the case of two variables (but this restriction is relevant in one step of the proof only).

Usually Harnack's inequality is formulated in terms of nonnegative solutions (see e.g. [4), which is no restriction if $u$ is a solution if and only if $c \pm u$ is a solution. However, here we write it in the form

$$
\sup _{\mathbf{x} \in B\left(\mathbf{x}_{0}, \frac{1}{2} R\right)} u(\mathbf{x}) \leq \alpha \sup _{\mathbf{x} \in B\left(\mathbf{x}_{0}, R\right)} u(\mathbf{x})+(1-\alpha) \inf _{\mathbf{y} \in B\left(\mathbf{x}_{0}, \frac{1}{2} R\right)} u(\mathbf{y}),
$$

where $\alpha \in(0,1)$ in order to stress that it is a quantitative version of the strong maximum principle which says that if a local maximum is achieved at some point in the interior of the set $\Omega$, then the solution is locally a constant.

We say that $u$ is a viscosity solution if it is both a subsolution and a supersolution and that $u$ is a subsolution to $F=0$ in $\Omega$ if it is upper semicontinuous in $\Omega$ and for every $\mathbf{x}_{0} \in \Omega$ the following implication holds: If $\psi \in \mathcal{C}^{2}\left(\mathbb{R}^{d}\right)$ and $u(\mathbf{x}) \leq \psi(\mathbf{x})+u\left(\mathbf{x}_{0}\right)-\psi\left(\mathbf{x}_{0}\right), \mathbf{x} \in \Omega,\left|\mathbf{x}-\mathbf{x}_{0}\right|<\delta$, for some $\delta>0$, then $F\left(\mathbf{x}_{0}, u\left(\mathbf{x}_{0}\right), D \psi\left(\mathbf{x}_{0}\right), D^{2} \psi\left(\mathbf{x}_{0}\right)\right) \leq 0$. (Here $D \psi\left(\mathbf{x}_{0}\right)$ is the gradient of $\psi$ and $D^{2} \psi\left(\mathbf{x}_{0}\right)$ is the matrix with the second order partial derivatives of $\psi$ at $\mathbf{x}_{0}$ as elements.) Supersolutions are defined in a similar way with " $\leq$ " replaced by " $\geq$ ". For details, further information, references, and related results not discussed here, see e.g. 22 and 3. Since one wants to be sure that classical solutions are viscosity

Received by the editors August 21, 2008.

2000 Mathematics Subject Classification. Primary 35J60.

Key words and phrases. Harnack's inequality, viscosity solution.

(C)2009 American Mathematical Society Reverts to public domain 28 years from publication 
solutions, one has to assume that $F$ is nonincreasing in its last argument (with the natural ordering for symmetric matrices).

In certain cases, such as the equation $-\Delta_{p} u=0$ where $p \in(d, \infty]$ (and $d$ is the dimension), one gets a Harnack inequality as above for subsolutions (and a corresponding one for supersolutions with the inequality reversed and sup and inf interchanged). This follows from the fact that the function $\left|\mathbf{x}-\mathbf{x}_{0}\right|^{\gamma}$ is a supersolution to $-\Delta_{p} u=0$ in $\left\{\mathbf{x} \mid \mathbf{x} \neq \mathbf{x}_{0}\right\}$ provided $\gamma \in\left(0, \frac{p-d}{p-1}\right)$. This is related to the fact that one can choose $\eta<2$ in assumption (iii) in Theorem 1 below. But in other cases such as $-\Delta_{p} u=0$ with $1<p \leq d$, one cannot have a Harnack inequality for subsolutions only. In this paper we consider subsolutions that are continuous and satisfy the strong minimum property. Clearly one gets a Harnack inequality in the opposite sense for continuous supersolutions satisfying the strong maximum principle under symmetrical assumptions.

We let $B\left(\mathbf{x}_{0}, r\right)=\left\{\mathbf{x} \in \mathbb{R}^{d}|| \mathbf{x}-\mathbf{x}_{0} \mid<r\right\}$ where $|\cdot|$ denotes the Euclidean norm, by $\mathcal{S}(d)$ we denote the set of all $d \times d$ symmetric matrices, and for $A$ and $B \in \mathcal{S}(d)$ we write $A \leq B$ if $\langle\mathbf{x},(A-B) \mathbf{x}\rangle \leq 0$ for all $\mathbf{x} \in \mathbb{R}^{d}$ and $A<B$ if $\langle\mathbf{x},(A-B) \mathbf{x}\rangle<0$ for all $\mathbf{x} \in \mathbb{R}^{d} \backslash\{\mathbf{0}\}$, where $\langle\cdot, \cdot\rangle$ denotes the standard inner product in $\mathbb{R}^{d}$. If $\mathbf{n} \in \mathbb{R}^{d}$, then $\mathbf{n} \otimes \mathbf{n}$ denotes the matrix with elements $\mathbf{n}_{j} \mathbf{n}_{k}$. If $A \in \mathcal{S}(d)$ we denote by $\sigma(A)$ the set of eigenvalues of $A$ (which is contained in $\mathbb{R}$ ).

Theorem 1. Assume that $d=2$ and that

(i) $\Omega \subset \mathbb{R}^{d}$ is open and nonempty.

(ii) $F: \Omega \times \mathbb{R} \times \mathbb{R}^{d} \times \mathcal{S}(d) \rightarrow[-\infty, \infty]$ is nonincreasing in its fourth variable.

(iii) For every compact subset $\mathcal{B} \subset \Omega$ and every $\lambda_{0}>0$ there is a number $\eta=\eta\left(\lambda_{0}, \mathcal{B}\right)>0$ such that

$$
F\left(\mathbf{x}, u(\mathbf{x}), \mathbf{p}, \lambda|\mathbf{p}| I-\frac{\lambda \eta}{|\mathbf{p}|} \mathbf{p} \otimes \mathbf{p}\right)>0
$$

for all $\mathbf{x} \in \mathcal{B}, \mathbf{p} \neq 0$, and $\lambda \geq \lambda_{0}$.

(iv) $u: \Omega \rightarrow \mathbb{R}$ is a viscosity subsolution to the equation $F\left(\mathbf{x}, u, D u, D^{2} u\right)=0$ in $\Omega$.

(v) $u$ is continuous and has no local minimum points in $\Omega$ in which $u$ is not locally a constant.

Then for each compact subset $\mathcal{B} \subset \Omega$ there is a number $\alpha \in(0,1)$ (independent of u) so that for each ball $B\left(\mathbf{x}_{0}, R\right) \subset \mathcal{B}$,

$$
\sup _{\mathbf{x} \in B\left(\mathbf{x}_{0}, \frac{1}{2} R\right)} u(\mathbf{x}) \leq \alpha \sup _{\mathbf{x} \in B\left(\mathbf{x}_{0}, R\right)} u(\mathbf{x})+(1-\alpha) \inf _{\mathbf{x} \in B\left(\mathbf{x}_{0}, \frac{1}{2} R\right)} u(\mathbf{x}) .
$$

Another way to formulate the result is to observe that (iii) can be used to establish the strong maximum principle (see e.g. 1]), although somewhat weaker assumptions suffice. Similarly with corresponding assumptions the strong minimum principle can be established so that assumption ( $\mathbb{\nabla}$ ) is not needed and we can get a two-sided Harnack's inequality as well.

Corollary 2. Assume that $d=2$ and that

(i) $\Omega \subset \mathbb{R}^{d}$ is open and nonempty.

(ii) $F: \Omega \times \mathbb{R} \times \mathbb{R}^{d} \times \mathcal{S}(d) \rightarrow[-\infty, \infty]$ is nonincreasing in its fourth variable. 
(iii) For every compact subset $\mathcal{B} \subset \Omega$ and every $\lambda_{0}>0$ there is a number $\eta=\eta\left(\lambda_{0}, \mathcal{B}\right)>0$ such that

$$
\begin{aligned}
F\left(\mathbf{x}, u(\mathbf{x}), \mathbf{p}, \lambda|\mathbf{p}| I-\frac{\lambda \eta}{|\mathbf{p}|} \mathbf{p} \otimes \mathbf{p}\right) & >0 \\
& \text { and } \quad F\left(\mathbf{x}, u(\mathbf{x}), \mathbf{p},-\lambda|\mathbf{p}| I+\frac{\lambda \eta}{|\mathbf{p}|} \mathbf{p} \otimes \mathbf{p}\right)<0
\end{aligned}
$$

for all $\mathbf{x} \in \mathcal{B}, \mathbf{p} \neq 0$, and $\lambda \geq \lambda_{0}$.

(iv) $u: \Omega \rightarrow \mathbb{R}$ is a viscosity solution to the equation $F\left(\mathbf{x}, u, D u, D^{2} u\right)=0$ in $\Omega$.

Then for each compact subset $\mathcal{B} \subset \Omega$ there is a number $\alpha \in(0,1)$ so that for each ball $B\left(\mathbf{x}_{0}, R\right) \subset \mathcal{B}$,

$$
\begin{aligned}
& \sup _{\mathbf{x} \in B\left(\mathbf{x}_{0}, \frac{1}{2} R\right)} u(\mathbf{x}) \leq \alpha \sup _{\mathbf{x} \in B\left(\mathbf{x}_{0}, R\right)} u(\mathbf{x})+(1-\alpha) \inf _{\mathbf{x} \in B\left(\mathbf{x}_{0}, \frac{1}{2} R\right)} u(\mathbf{x}), \\
& \inf _{\mathbf{x} \in B\left(\mathbf{x}_{0}, \frac{1}{2} R\right)} u(\mathbf{x}) \geq \alpha \inf _{\mathbf{x} \in B\left(\mathbf{x}_{0}, R\right)} u(\mathbf{x})+(1-\alpha) \sup _{\mathbf{x} \in B\left(\mathbf{x}_{0}, \frac{1}{2} R\right)} u(\mathbf{x}) .
\end{aligned}
$$

In the proof of Theorem 1 we need the following lemma, which we formulate and prove in dimension $d \geq 2$.

Lemma 3. Assume that $d \geq 2$ and that

(i) $\Omega \subset \mathbb{R}^{d}$ is open and nonempty.

(ii) $F: \Omega \times \mathbb{R} \times \mathbb{R}^{d} \times \mathcal{S}(d) \rightarrow[-\infty, \infty]$ is nonincreasing in its fourth variable.

(iii) $A \in \mathcal{S}(d)$ and $A>0$.

(iv) $\Omega_{*} \subset \Omega \cap\left\{\mathbf{x} \mid R_{1}^{2} \leq\left\langle\mathbf{x}-\mathbf{x}_{*}, A\left(\mathbf{x}-\mathbf{x}_{*}\right)\right\rangle \leq R_{2}^{2}\right\}$ is closed, where $0<R_{1}<$ $R_{2}<\infty$.

(v) $u: \Omega \rightarrow[-\infty, \infty)$ is a subsolution to the equation $F\left(\mathbf{x}, u, D u, D^{2} u\right)=0$ in $\Omega$.

(vi) There are constants $-\infty<M_{1}<M_{2}<\infty$ so that $u(\mathbf{x}) \leq M_{1}$ when $\mathbf{x} \in \partial \Omega_{*} \cap\left\{\mathbf{x} \mid\left\langle\mathbf{x}-\mathbf{x}_{*}, A\left(\mathbf{x}-\mathbf{x}_{*}\right)\right\rangle<R_{2}^{2}\right\}$ and $u(\mathbf{x}) \leq M_{2}$ when $\mathbf{x} \in$ $\partial \Omega_{*} \cap\left\{\mathbf{x} \mid\left\langle\mathbf{x}-\mathbf{x}_{*}, A\left(\mathbf{x}-\mathbf{x}_{*}\right)\right\rangle=R_{2}^{2}\right\}$.

(vii) For every compact subset $\mathcal{B} \subset \Omega$ and every $\lambda>0$ there is a number $\eta=$ $\eta(\lambda, \mathcal{B})>0$ such that

$$
F\left(\mathbf{x}, u(\mathbf{x}), \mathbf{p}, \lambda|\mathbf{p}| I-\frac{\lambda \eta}{|\mathbf{p}|} \mathbf{p} \otimes \mathbf{p}\right)>0
$$

for all $\mathbf{x} \in \mathcal{B}$ and $\mathbf{p} \neq 0$.

Then

$$
\begin{aligned}
u(\mathbf{x}) & \leq \frac{M_{2}\left(R_{1}^{-2 \gamma}-R^{-2 \gamma}\right)+M_{1}\left(R^{-2 \gamma}-R_{2}^{-2 \gamma}\right)}{R_{1}^{-2 \gamma}-R_{2}^{-2 \gamma}}, \\
R & =\sqrt{\left\langle\mathbf{x}-\mathbf{x}_{*}, A\left(\mathbf{x}-\mathbf{x}_{*}\right)\right\rangle}, \quad \mathbf{x} \in \Omega_{*},
\end{aligned}
$$

where $\gamma>0$ satisfies $\gamma \geq \frac{\max \sigma(A)}{2 \min \sigma(A)} \eta\left(\frac{\max \sigma(A)}{\rho \sqrt{\min \sigma(A)}}, \Omega_{*}\right)-1$ for all $\rho \in\left(R_{1}, R_{2}\right)$.

A simple case where this lemma can be applied is where $A=I$ and $\Omega_{*}=\{\mathbf{x} \mid$ $\left.R_{1}^{2} \leq\left|\mathbf{x}-\mathbf{x}_{*}\right|^{2} \leq R_{2}^{2}\right\}$, and the conclusion is that if $u$ is "small" when $\left|\mathbf{x}-\mathbf{x}_{*}\right|=R_{1}$, then it is not "too big" in $\left\{\mathbf{x}\left|R_{1}^{2} \leq\right| \mathbf{x}-\left.\mathbf{x}_{*}\right|^{2} \leq R_{2}^{2}\right\}$ for $R_{1}<\rho<R_{2}$. However, this requires (unless one could take $\gamma<0$ ) that $R_{1}$ is not "too small". Thus the 
main strategy in the proof of Theorem 1 is to find sufficiently "large" sets where the solution is "small".

\section{Proofs}

Proof of Lemma 3. Let $\varphi(\mathbf{x})=-a\left\langle\mathbf{x}-\mathbf{x}_{*}, A\left(\mathbf{x}-\mathbf{x}_{*}\right)\right\rangle^{-\gamma}+b$, where $a=\frac{M_{2}-M_{1}}{R_{1}^{-2 \gamma}-R_{2}^{-2 \gamma}}$ and $b=\frac{M_{2} R_{1}^{-2 \gamma}-M_{1} R_{2}^{-2 \gamma}}{R_{1}^{-2 \gamma}-R_{2}^{-2 \gamma}}$. Thus we see that when $\left\langle\mathbf{x}-\mathbf{x}_{*}, A\left(\mathbf{x}-\mathbf{x}_{*}\right)\right\rangle \geq R_{1}^{2}$ we have $\varphi(\mathbf{x}) \geq M_{1}$ and when $\left\langle\mathbf{x}-\mathbf{x}_{*}, A\left(\mathbf{x}-\mathbf{x}_{*}\right)\right\rangle=R_{2}^{2}$ we have $\varphi(\mathbf{x})=M_{2}$. Thus it follows from our assumptions that $u(\mathbf{x}) \leq \varphi(\mathbf{x})$ for $\mathbf{x} \in \partial \Omega_{*}$. The claim of the lemma is that this inequality holds for all $\mathbf{x} \in \Omega_{*}$, so if this is not the case, then the maximum of $u-\varphi$ in $\Omega_{*}$ must be achieved in an interior point. Assume that this point is $\mathbf{x}_{1}$. Since $u$ is a subsolution we have $F\left(\mathbf{x}_{1}, u\left(\mathbf{x}_{1}\right), D \varphi\left(\mathbf{x}_{1}\right), D^{2} \varphi\left(\mathbf{x}_{1}\right)\right) \leq 0$, and once we have shown that we in fact must have $F\left(\mathbf{x}_{1}, u\left(\mathbf{x}_{1}\right), D \varphi\left(\mathbf{x}_{1}\right), D^{2} \varphi\left(\mathbf{x}_{1}\right)\right)>0$ we get the desired conclusion (thus here we just use comparison between a subsolution and a twice continuously differentiable strict supersolution).

Now

$$
D \varphi\left(\mathbf{x}_{1}\right)=2 a \gamma\left\langle\mathbf{x}_{1}-\mathbf{x}_{*}, A\left(\mathbf{x}_{1}-\mathbf{x}_{*}\right)\right\rangle^{-\gamma-1} A\left(\mathbf{x}_{1}-\mathbf{x}_{*}\right)
$$

and

$$
\begin{array}{r}
D^{2} \varphi\left(\mathbf{x}_{1}\right)=-4 a \gamma(\gamma+1)\left\langle\mathbf{x}_{1}-\mathbf{x}_{*}, A\left(\mathbf{x}_{1}-\mathbf{x}_{*}\right)\right\rangle^{-\gamma-2}\left(A\left(\mathbf{x}_{1}-\mathbf{x}_{*}\right)\right) \otimes\left(A\left(\mathbf{x}_{1}-\mathbf{x}_{*}\right)\right) \\
+2 a \gamma\left\langle\mathbf{x}_{1}-\mathbf{x}_{*}, A\left(\mathbf{x}_{1}-\mathbf{x}_{*}\right)\right\rangle^{-\gamma-1} A .
\end{array}
$$

Let $\left\langle\mathbf{x}_{1}-\mathbf{x}_{*}, A\left(\mathbf{x}_{1}-\mathbf{x}_{*}\right)\right\rangle=\rho_{1}^{2}$. Then we have $\left|A\left(\mathbf{x}_{1}-\mathbf{x}_{*}\right)\right| \geq \rho_{1} \sqrt{\min \sigma(A)}$. Thus we see that if we choose $\lambda=\frac{\max \sigma(A)}{\rho_{1} \sqrt{\min \sigma(A)}}$, we have

$$
2 a \gamma\left\langle\mathbf{x}_{1}-\mathbf{x}_{*}, A\left(\mathbf{x}_{1}-\mathbf{x}_{*}\right)\right\rangle^{-\gamma-1} A \leq \lambda\left|D \varphi\left(\mathbf{x}_{1}\right)\right| I .
$$

Similarly, a calculation shows that

$$
\begin{aligned}
&-4 a \gamma(\gamma+1)\left\langle\mathbf{x}_{1}-\mathbf{x}_{*}, A\left(\mathbf{x}_{1}-\mathbf{x}_{*}\right)\right\rangle^{-\gamma-2}\left(A\left(\mathbf{x}_{1}-\mathbf{x}_{*}\right)\right) \otimes\left(A\left(\mathbf{x}_{1}-\mathbf{x}_{*}\right)\right) \\
&=-\frac{2\left|A\left(\mathbf{x}_{1}-\mathbf{x}_{*}\right)\right|}{\left\langle\mathbf{x}_{1}-\mathbf{x}_{*}, A\left(\mathbf{x}_{1}-\mathbf{x}_{*}\right)\right\rangle} \frac{\gamma+1}{\left|D \varphi\left(\mathbf{x}_{1}\right)\right|} D \varphi\left(\mathbf{x}_{1}\right) \otimes D \varphi\left(\mathbf{x}_{1}\right) \\
& \leq-\frac{2 \sqrt{\min \sigma(A)}(\gamma+1)}{\rho_{1}} \frac{1}{\left|D \varphi\left(\mathbf{x}_{1}\right)\right|} D \varphi\left(\mathbf{x}_{1}\right) \otimes D \varphi\left(\mathbf{x}_{1}\right) \\
&=-\frac{2(\gamma+1) \min \sigma(A)}{\max \sigma(A)} \frac{\lambda}{\left|D \varphi\left(\mathbf{x}_{1}\right)\right|} D \varphi\left(\mathbf{x}_{1}\right) \otimes D \varphi\left(\mathbf{x}_{1}\right) .
\end{aligned}
$$

Now our choice of $\gamma$ guarantees that

$$
D^{2} \varphi\left(\mathbf{x}_{1}\right) \leq \lambda\left|D \varphi\left(\mathbf{x}_{1}\right)\right| I-\frac{\eta\left(\lambda, \Omega_{*}\right) \lambda}{\left|D \varphi\left(\mathbf{x}_{1}\right)\right|} D \varphi\left(\mathbf{x}_{1}\right) \otimes D \varphi\left(\mathbf{x}_{1}\right),
$$

and from our assumption (viil) we get the desired contradiction.

Proof of Theorem 1. The strong minimum principle contained in hypothesis (v) implies that $\inf _{\mathbf{y} \in B\left(\mathbf{x}_{0}, \frac{1}{2} R\right)} u(\mathbf{y})$ is achieved at some point $\mathbf{x}_{1}$ with $\left|\mathbf{x}_{1}-\mathbf{x}_{0}\right|=\frac{1}{2} R$. We may without loss of generality choose the coordinate system so that $\mathbf{x}_{1}-\mathbf{x}_{0}$ lies in the direction of the positive $x$-axis. Let $a=\frac{1}{4} R$ and $b=\frac{\sqrt{15}}{4} a$. Furthermore, let $H_{1}$ be the open rectangle with corners at $\mathbf{x}_{1}+(0, \pm a)$ and $\mathbf{x}_{1}+(b, \pm a)$, let $H_{2}$ be the open rectangle with corners at $\mathbf{x}_{1}+( \pm a, b)$ and $\mathbf{x}_{1}+( \pm a, 0)$, and finally let $H_{3}$ be the open rectangle with corners at $\mathbf{x}_{1}+( \pm a,-b)$ and $\mathbf{x}_{1}+( \pm a, 0)$. If for each $k \in\{1,2,3\}$ 
there is a connected open component of $H_{k} \cap\left\{\mathbf{x} \mid u(\mathbf{x})>\inf _{\mathbf{y} \in B\left(\mathbf{x}_{0}, \frac{1}{2} R\right)} u(\mathbf{y})\right\}$ whose closure intersects both the shorter sides of the rectangle $H_{k}$, then we can construct a closed simple curve in the interior of the square with corners at $\mathbf{x}_{1}+( \pm b, \pm b)$ so that $u(\mathbf{x}) \geq \inf _{\mathbf{y} \in B\left(\mathbf{x}_{0}, \frac{1}{2} R\right)} u(\mathbf{y})$ when $\mathbf{x}$ is on the curve with strict inequality at some points. (Here the assumption that the dimension is two is crucial, and the argument is somewhat simpler when we take $b<a$.) But this contradicts the strong minimum principle, and thus there is a number $k \in\{1,2,3\}$ so that there is no such connected open component for this number $k$.

Let

$$
\mathbf{x}_{2}=\mathbf{x}_{1}+ \begin{cases}\left(\frac{b}{2},-2 a\right), & k=1 \\ \left(2 a, \frac{b}{2}\right), & k=2, \\ \left(2 a,-\frac{b}{2}\right), & k=3\end{cases}
$$

Now we apply Lemma 3 with $\mathbf{x}_{*}=\mathbf{x}_{2}, R_{1}=\frac{1}{4} a$, and $R_{2}=a$; and if $k=1$ we take $A=\left[\begin{array}{cc}4 & 0 \\ 0 & \frac{1}{16}\end{array}\right]$, and in the other cases we take $A=\left[\begin{array}{cc}\frac{1}{16} & 0 \\ 0 & 4\end{array}\right]$. We let

$$
\Omega_{*}=\left\{\mathbf{x} \mid\left\langle\mathbf{x}-\mathbf{x}_{*}, A\left(\mathbf{x}-\mathbf{x}_{*}\right)\right\rangle \leq R_{2}^{2}\right\} \cap\left(\overline{\Omega_{k}} \cup \overline{Q_{k}}\right) .
$$

In Figure 1 the shaded regions are possible sets $\Omega_{*}$ in the cases $k=1$ and $k=2$, respectively. In this definition $\overline{\Omega_{k}}$ is the closure of $\Omega_{k}$, which is the union of the connected open components of $H_{k} \cap\left\{\mathbf{x} \mid u(\mathbf{x})>\inf _{\mathbf{y} \in B\left(\mathbf{x}_{0}, \frac{1}{2} R\right)} u(\mathbf{y})\right\}$ whose closures have nonempty intersections with the line segment from $\mathbf{x}_{1}+(0, a)$ to $\mathbf{x}_{1}+(b, a)$ if $k=1$, the line segment from $\mathbf{x}_{1}+(-a, 0)$ to $\mathbf{x}_{1}+(-a, b)$ if $k=2$, and the line segment from $\mathbf{x}_{1}+(-a, 0)$ to $\mathbf{x}_{1}+(-a,-b)$ if $k=3$. The set $Q_{k}$ is the rectangle with corners at $\mathbf{x}_{1}+(0, a), \mathbf{x}_{1}+(0,2 a), \mathbf{x}_{1}+(b, 2 a)$, and $\mathbf{x}_{1}+(b, a)$ when $k=1$; the rectangle with corners at $\mathbf{x}_{1}+(-a, 0), \mathbf{x}_{1}+(-2 a, 0), \mathbf{x}_{1}+(-2 a, b)$, and $\mathbf{x}_{1}+(-a, b)$ when $k=2$; and finally the rectangle with corners at $\mathbf{x}_{1}+(-a, 0), \mathbf{x}_{1}+(-2 a, 0)$, $\mathbf{x}_{1}+(-2 a,-b)$, and $\mathbf{x}_{1}+(-a,-b)$ when $k=3$. Our choice of $a$ guarantees that
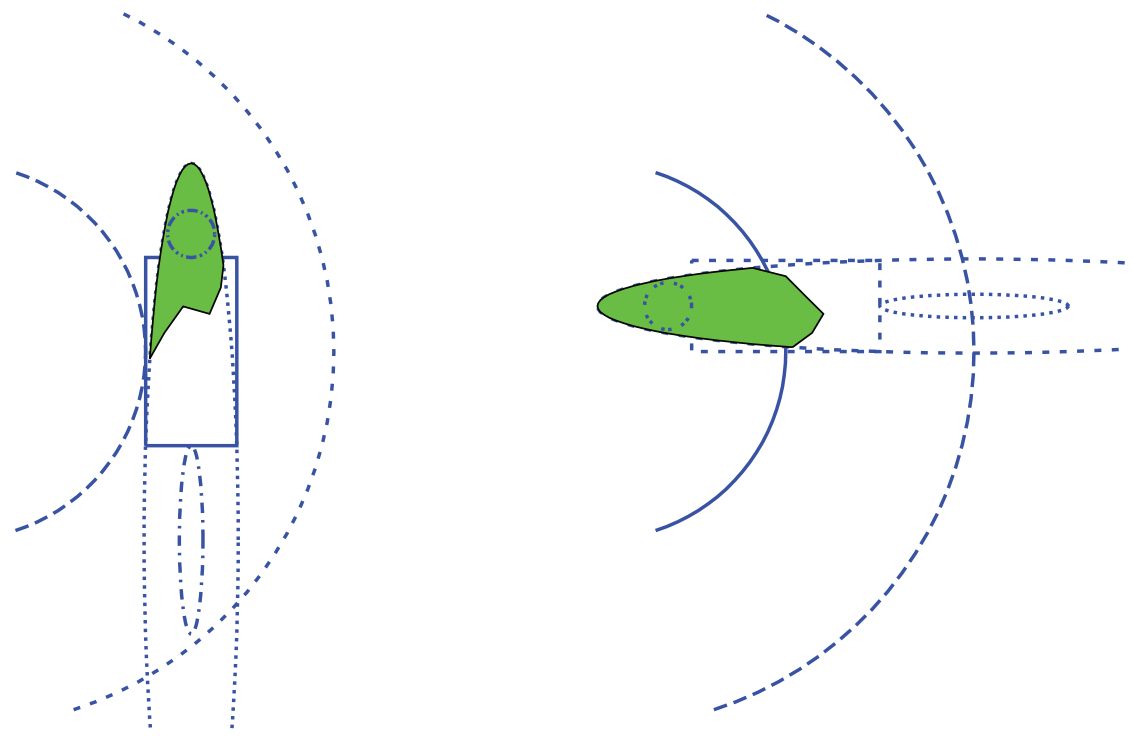

FiguRE 1 
$\Omega_{*} \subset B\left(\mathbf{x}_{0}, R\right)$, and we conclude from our choice of $k$ (and $b$ ) that hypothesis (vil) in Lemma 3 with $M_{1}=\inf _{\mathbf{y} \in B\left(\mathbf{x}_{0}, \frac{1}{2} R\right)} u(\mathbf{y})$ and $M_{2}=\sup _{\mathbf{y} \in B\left(\mathbf{x}_{0}, R\right)} u(\mathbf{y})$ is satisfied since the set $\left\{\mathbf{x} \mid\left\langle\mathbf{x}-\mathbf{x}_{*}, A\left(\mathbf{x}-\mathbf{x}_{*}\right)\right\rangle \leq R_{2}^{2}\right\}$ does not intersect the longer sides of the rectangle $H_{k}$. If we now choose

$$
\mathbf{x}_{3}=\mathbf{x}_{1}+ \begin{cases}\left(\frac{b}{2}, \frac{5 a}{4}\right), & k=1, \\ \left(-\frac{5 a}{4}, \frac{b}{2}\right), & k=2, \\ \left(-\frac{5 a}{4},-\frac{b}{2}\right), & k=3,\end{cases}
$$

then we conclude that $B\left(\mathbf{x}_{3}, \frac{a}{4}\right) \subset \Omega_{*}$ and $\left\langle\mathbf{x}-\mathbf{x}_{*}, A\left(\mathbf{x}-\mathbf{x}_{*}\right)\right\rangle \leq \frac{1}{2} a^{2}$ when $\mathbf{x} \in$ $B\left(\mathbf{x}_{3}, \frac{a}{4}\right)$. Thus we conclude from Lemma 3 that there is a number $\alpha_{3} \in(0,1)$ independent of $R$ (but note that $R$ is bounded from above since $B\left(\mathbf{x}_{0}, R\right) \subset \mathcal{B}$ ) such that

$$
u(\mathbf{x}) \leq \alpha_{3} \sup _{\mathbf{x} \in B\left(\mathbf{x}_{0}, R\right)} u(\mathbf{x})+\left(1-\alpha_{3}\right) \inf _{\mathbf{y} \in B\left(\mathbf{x}_{0}, \frac{1}{2} R\right)} u(\mathbf{y}), \quad \mathbf{x} \in B\left(\mathbf{x}_{3}, R_{3}\right),
$$

where $R_{3}=\frac{1}{16} R$.

Now we can repeatedly use Lemma 3 for $j=4,5,6,7$, this time with $A=I$, $\mathbf{x}_{*}=\mathbf{x}_{j-1}, R_{1}=R_{j-1}, R_{2}=R-\left|\mathbf{x}_{j-1}-\mathbf{x}_{0}\right|, \Omega_{*}=\left\{\mathbf{x}\left|R_{1} \leq\right| \mathbf{x}-\mathbf{x}_{*} \mid \leq\right.$ $\left.R_{2}\right\}, M_{1}=\alpha_{j-1} \sup _{\mathbf{y} \in B\left(\mathbf{x}_{0}, R\right)} u(\mathbf{y})+\left(1-\alpha_{j-1}\right) \inf _{\mathbf{y} \in B\left(\mathbf{x}_{0}, \frac{1}{2} R\right)} u(\mathbf{y})$, and with $M_{2}=$ $\sup _{\mathbf{y} \in B\left(\mathbf{x}_{0}, R\right)} u(\mathbf{y})$ to conclude that there is a number $\alpha_{j} \in(0,1)$ so that

$$
u(\mathbf{x}) \leq \alpha_{j} \sup _{\mathbf{y} \in B\left(\mathbf{x}_{0}, R\right)} u(\mathbf{y})+\left(1-\alpha_{j}\right) \inf _{\mathbf{y} \in B\left(\mathbf{x}_{0}, \frac{1}{2} R\right)} u(\mathbf{y}), \quad \mathbf{x} \in B\left(\mathbf{x}_{j}, R_{j}\right),
$$

where

and

$$
\mathbf{x}_{j}=\mathbf{x}_{j-1}-\min \left\{\frac{R-\left|\mathbf{x}_{j-1}-\mathbf{x}_{0}\right|}{2\left|\mathbf{x}_{j-1}-\mathbf{x}_{0}\right|}, 1\right\}\left(\mathbf{x}_{j-1}-\mathbf{x}_{0}\right)
$$

$$
R_{j}=\min \left\{\frac{3}{4}\left(R-\left|\mathbf{x}_{j-1}-\mathbf{x}_{0}\right|-\left|\mathbf{x}_{j}-\mathbf{x}_{j-1}\right|\right), \frac{1}{2} R\right\} .
$$

Since $\left|\mathbf{x}_{3}-\mathbf{x}_{0}\right| \leq \frac{3}{4} R$, a calculation shows that we have $\mathbf{x}_{7}=\mathbf{x}_{0}$ and $R_{7}=\frac{1}{2} R$. This completes the proof.

\section{REFERENCES}

1. G. Barles and J. Busca. Existence and comparison results for fully nonlinear degenerate elliptic equations without zeroth-order term. Comm. Partial Differential Equations, 26(11-12):23232337, 2001. MR 1876420(2002k:35078)

2. L.A. Caffarelli and X. Cabré. Fully nonlinear elliptic equations. Volume 43 of American Mathematical Society Colloquium Publications. American Mathematical Society, Providence, RI, 1995. MR1351007 (96h:35046)

3. M.G. Crandall, H. Ishii, and P.L. Lions. User's guide to viscosity solutions of second order partial differential equations. Bull. Amer. Math. Soc. (N.S.), 27:1-67, 1992. MR1118699 $(92 \mathrm{j}: 35050)$

4. D. Gilbarg and N.S. Trudinger. Elliptic partial differential equations of second order. Classics in Mathematics. Springer-Verlag, Berlin, 2001. Reprint of the 1998 edition. MR1814364 (2001k:35004)

Department of Mathematics and Systems Analysis, Helsinki University of TechnolOGY, P.O. Box 1100, FIN-02015 TKK, Finland

E-mail address: gustaf.gripenberg@tkk.fi 